\title{
Optimal End-to-End Transmission Control of Satellite Networks with Long Delay and High Bit Error Rate
}

\author{
Liang Zong ${ }^{1}$, Yudan Ning ${ }^{2 *}$, Yong Bai ${ }^{3}$, Chenglin Zhao ${ }^{1}$ and Gaofeng Luo ${ }^{1}$ \\ ${ }^{1}$ College of Information Engineering, Shaoyang University, Shaoyang 422000, China \\ ${ }^{2}$ Department of Information Technology and Creativity, Shaoyang Polytechnic, Shaoyang 422000, China \\ ${ }^{3}$ School of Information and Communication Engineering, Hainan University, Haikou 570228, China
}

Received 2 October 2020; Accepted 19 December 2020

\begin{abstract}
Satellite networks have the characteristics of long transmission delay, high bit error rate (BER), and asymmetric wide band, which seriously influence the performance of traditional TCP/IP networks. In order to adapt to long delay and high BER of satellite networks, a transmission control algorithm was proposed in this study. The amount of data transmission was enhanced to fill the high bandwidth-delay product of satellite links. The transmission factor was used to estimate available bandwidth in slow start. The reason for data loss was evaluated by the data backlog value in congestion avoidance. In this way, the threshold was increased in fast recovery, and the data transmission was improved in a short time. Moreover, the value of the congestion window in slow start and congestion avoidance was analyzed. And the threshold was adjusted by different network conditions. Results demonstrate that the proposed algorithm effectively improves the throughput of satellite links by $57.14 \%$ compared with TCP New Reno and also distinguishes different data losses. These findings indicate the transmission mechanism can take lower download response time. The proposed algorithm provides a good prospect to improve the transmission performance of satellite networks.
\end{abstract}

Keywords: Transmission control, Satellite networks, TCP, High bit error rate

\section{Introduction}

Satellite networks with the characteristics of global coverage, broadband flexibility, reliability, and multicast are extremely important in a cross-regional communication environment, especially in remote areas without infrastructure [1]. For example, the access of shipborne satellite communication equipment and satellite networks may be the only choice in an ocean far away from the mainland. Satellite networks were accessed to the global Internet in the 1970s. Owing to the small link capacity of satellite networks, minimal attention was paid to the performance of satellite network transmission. However, with the development of satellite communication technology, satellite networks with high frequency and bandwidth have become the mainstream of satellite communications. New challenges have been posed to the congestion control of satellite networks, which is influenced by long transmission delay, asymmetric uplink and downlink bandwidth, and the high bit error rate of links.

With the rise of mobile Internet, increased requirements have been raised for accessing the Internet anytime and anywhere. The satellite network, as a supplement to a ground network, has become an important part of the Internet. Nevertheless, its long transmission delay, high BER, and asymmetric broadband [2] affect the performance of the traditional TCP/IP for Internet networks. Meanwhile, great challenges are posed to the study of the transmission performance of satellite networks [3]. To improve the

*E-mail address: yudan_ning@163.com

ISSN: 1791-2377@ 2020 School of Science, IHU. All rights reserved. doi:10.25103/jestr.136.11 transmission performance of satellite networks, scholars have conducted numerous studies to weaken the effects of the long propagation delay, high bit error rate, and broadband asymmetry of satellite networks [4-7]. Existing studies of the transmission performance of satellite networks focus on the influence of a single factor [8-10] and have achieved remarkable results.

Under the influences of multiple factors, such as long delay and high BER, it is challenging for improving the transmission performance of satellite networks. Therefore, how to improve the performance of satellite network transmission under the influences of multiple factors is an urgent problem to be solved.

This study proposed a transmission control algorithm that can adapt to the long delay and high BER of satellite networks. The algorithm weakens the effects of long delay and high BER on transmission performance. The transmission amount of initial data is increased under the influence of long time delay; thus, the high bandwidth-delay product of satellite networks can be utilized effectively. The reason for data loss under a high BER can be judged by calculating the data backlog value in satellite networks. Accordingly, the threshold value is timely adjusted in the rapid recovery stage, and the data transmission amount is adjusted in different data packet loss environments.

\section{State of the art}

Scholars have conducted many studies to improve the transmission performance of satellite networks. For instance, Bisu [11, 12] proposed an accurate method for measuring the end-to-end (E2E) delay of IP in satellite networks. An 
integrated test platform was established to analyze the performance of different schemes in two geostationary satellite networks. The results showed that IP packet process and transmission occupy at least half of the delay time. However, no effective solution has been determined.

Saedi [13] proposed a scheme to adjust the threshold dynamically by the round-trip time (RTT). The RTT was smoothed to compare the minimum RTT value and evaluated congestion in networks, thereby conducting random or congestion loss processing for the lost packets. Nonetheless, no effective solution exists for the long delay of satellite networks. Liu [14] adjusted the transmission rate of the sender in accordance with packet feedback information. The transmission rate decline caused by the high BER in the wireless communication environment can be alleviated by adjusting the transmission rate and the size of the congestion window (CWND). The scheme can effectively maintain a high transmission rate under high BER. Although the method can adjust the transmission rate by using feedback information, it fails to solve the problem of a large amount of data transmitted by satellite networks with the long delay.

In recent years, machine learning and deep learning have exerted great effects on information technology. Nie [15] introduced the reinforcement learning idea into congestion control. Short and long flow services were studied, and the value of the initial window in slow start and the value of CWND in congestion avoidance were adjusted to improve the performance of the transmission control protocol. The study developed a new direction for studying network congestion control by machine learning and deep learning. However, whether the scheme can adapt to the satellite network needs further study. Polese [16] performed a comprehensive analysis of the combination of machine learning and congestion control. The feasibility of machine learning in the study of congestion control was confirmed, and multipath transmission was further discussed. Although great achievements have been achieved in the field of machine learning in recent years, how to combine the characteristics of satellite networks with the establishment of machine-learning models is still a challenging field.

Considering the success of machine learning, many scholars have turned to machine learning for network congestion control. Based on the study of machine learning and congestion control, Zhang [17] investigated the E2E congestion control of machine learning. Evidently, combining machine learning and congestion control is beneficial to improve the transmission performance of networks. Nevertheless, for the long delay and high BER of wireless networks, especially satellite networks, no effective method is available for establishing an effective machinelearning model. Li [18] proposed a boost model for satellite network and analyzed the loss of different packets. The reason for data loss can be effectively distinguished by classifying the model, in combination with the feedback information of Explicit Congestion Notification (ECN). Hence, a correct judgment basis can be provided for adjusting CWND. However, no solution to the long delay of satellite networks exists.

In terms of one-way delay, data transmission and website download time on the performance-enhancing proxy (PEP) scheme, Deutschmann [19] compared the performance of three major European satellite service providers. Nonetheless, PEP cannot adapt to the transmission protocol scheme among different devices. Considering the wide application of 5G mobile communications, Giambene [20] proposed architecture of $5 \mathrm{G}$ access satellite networks. In accordance with the network architecture, a scheme based on network coding was developed, and a good transmission effect was achieved in a multipath environment of satellite network. The scheme application should be improved further, given that the combination of $5 \mathrm{G}$ communications and satellite networks.

Ishii [21] developed a new real-time rate control algorithm for the serious influence on postdisaster ground network infrastructure. A congestion control scheme suitable for a heterogeneous network environment was established by analyzing the characteristic of long transmission delay of satellite networks. This scheme can effectively improve the transmission rate of satellite links. However, no reliable solution to the data loss of satellite networks under a high BER exists. In accordance with the Hypertext Transfer Protocol version 2 developed by the Internet Engineering Task Force and World Wide Web Consortium, Secchi [22] investigated the performance of web applications accessing satellite networks and analyzed the influence of long delay on satellite networks. Methods for improving network transmission performance were also determined. Nevertheless, the specific design of congestion control schemes needs to be further explored.

Quadrini [23] evaluated different methods for satellite networks in agent structures. An effective congestion control strategy was determined using a QUIC-based protocol to improve the transmission performance of satellite networks. However, the scheme was not inapplicable to E2E schemes for satellite networks. Utsumi [24] proposed a new delay analysis model in consideration of the good performance of TCP Hybla in satellite networks. The throughput of the new model in steady state in satellite networks was improved. The throughput and average transmission time can be effectively estimated in accordance with the estimated waiting time, thus enhancing the performance of satellite networks. Accurate information of the data packet should be confirmed to analyze the new model. In a satellite network with a high BER, random packet loss is possible, which challenges the accuracy of the model. TCP New Reno has achieved great success in wired networks, but its performance in satellite networks is poor. To analyze the upper and lower limits of TCP connection throughput, Liu [25] established a Markov chain model for TCP connection. Transition probability was proposed for the value of CWND in slow start and congestion avoidance phases. This scheme can evidently improve the transmission performance of random access to satellite networks. The scheme based on TCP New Reno cannot adapt to the high bandwidth-delay product in satellite networks.

The Internet of things (IoT) plays an increasingly important role in daily life, and how to combine satellites with IoT has aroused great interest. For example, Fraire [26] comprehensively reviewed and analyzed recent studies on Direct-To-Satellite IoT and discussed available physical layer technology designed for IoT satellite links. The applicability of medium access control and upper layer protocols was analyzed. The memory and endurance at the terminal are inferior to those at a wired terminal, which influences the continuous data transmission of satellites. Considering the link asymmetry of satellite networks, Guan [27] proposed a TCP Vegas forward congestion control algorithm. The algorithm determines whether a communication link is congested by measuring the forward delay time of data packets. In this way, the congestion control algorithm maintains a high throughput rate when the 
reverse link is congested and adapts to the bandwidth asymmetry of satellite networks. Although the algorithm utilizes real-time feedback information, no effective solution to long delay and high BER exists. $\mathrm{Xu}$ [28] proposed an improved MPTCP scheme for heterogeneous satellite networks, which returns acknowledge (ACK) through the shortest subpath to accelerate the growth of CWND. The available bandwidth is accurately estimated by confirming the arrival time of packets (ACK) to adjust the transmission rate. This scheme can effectively improve the E2E data throughput of heterogeneous satellite networks. Nonetheless, given that MPTCP is based on multipath transmission, its applicability is limited.

The aforementioned studies have focused on improving the transmission control protocol under the influence of a single factor of satellite networks and introduced machinelearning schemes into the transmission control algorithm of satellite networks. However, the study of the transmission control algorithm of satellite networks under the influences of multiple factors is scarce. Considering the long delay and high BER of satellite networks, this study establishes a method combining RTT and CWND control in accordance with the information of the ACK packet, which can greatly improve the transmission rate of satellite networks and effectively distinguish data packet loss. The study results provide a reference for improving the transmission performance of satellite networks.

The remainder of this study is organized as follows. Section 3 describes the new transmission scheme and establishes a calculation model for CWND. In Section 4, the transmission scheme is simulated and compared with different classic schemes. The new transmission scheme is determined to be able to adapt to the environment characteristics of long delay and high BER of satellite networks. Lastly, Section 5 summarizes the study and draws relevant conclusions.

\section{Methodology}

This study proposed a new transmission scheme to adapt to the long delay and high BER of satellite networks under the influences of multiple factors. The transmission amount of initial state under long delay was increased using a transmission factor to adjust the threshold value in the fast recovery phase. The data backlog value in satellite networks was calculated to determine the real reason for data loss. The performance of TCP in satellite links was improved by distinguishing different packet losses and changing the value of CWND.

\subsection{Transfer factor}

The new transmission scheme calculated the transmission factor $\rho$ and the change in the sender's correlation window by measuring RTT to control the size of CWND. The algorithm depended on a variable factor, which was measured in accordance with RTT, as shown as follows:

$$
\rho=R T T s \times 60
$$

where RTTs is the RTT measured by the sender in accordance with the data RTT. 60 is the minimum value set by the sender on the basis of the timeout retransmission timer, and its value range is $[1,60]$. During the algorithm implementation, the CWND of the sender was modified in accordance with the change factor.

\subsection{Detection of packet loss}

For the long delay in a satellite network, the transmission factor increased the amount of data transmission through the change factor, which improved the instantaneous throughput of the satellite network in the initial stage. However, the data loss caused by a high BER in the satellite link could not be distinguished. Similar to the traditional TCP New Reno, this algorithm considered that data loss was caused by congestion and directly started timeout retransmission to decrease the value of CWND of the sender. Consequently, the performance of data transmission was affected. The new scheme adopted the data backlog value similar to TCP Veno [29] and classified the backlog values in accordance with different adjustment thresholds. In this way, random packet loss in the wireless environment of the satellite network could be effectively distinguished, and the transmission performance could be improved.

Given that the feedback information received by the data sender in the satellite network was limited, data backlog value $N_{b a c k l o g}$ in the network could be calculated. The maximum rate $V_{\max }$ and the actual transmission rate $V_{\text {actual }}$ in the network were calculated, where cwnd, $R T T_{\min }$ and $R T T$ are the CWND, minimum RTT, and actual RTT values in connection, respectively.

$$
\begin{aligned}
& V_{\max }=c w n d / R T T_{\min } \\
& V_{\text {actual }}=c w n d / R T T
\end{aligned}
$$

The difference between them was as follows:

$$
V_{\max }-V_{\text {actual }}
$$

The value was not less than 0 , and the data backlog value $N_{\text {backlog }}$ in the network was calculated as follows:

$N_{\text {backlog }}=V_{\text {actual }} \times\left(R T T-R T T_{\text {min }}\right)=\left(V_{\text {max }}-V_{\text {actual }}\right) \times R T T_{\text {min }}$

In the above calculation, if the backlog value in the network increases or decreases, then the network state changes. When the backlog value in the network is greater than a certain threshold $\beta$, the congestion may cause excessive backlog data in the network; otherwise, limited backlog data exist in the network. Network congestion during packet loss can be judged in accordance with the backlog value.

\subsection{Slow start}

If the packet loss is detected, the data backlog value in the transmission network is judged. When $N_{\text {backlog }} \geq \beta$ and $N_{\text {backlog }}$ is large, the proposed enhanced algorithm can determine that the data loss is caused by congestion, and the CWND value of the traditional transmission control protocol is adopted. When $N_{\text {backlog }}<\beta$ and $N_{\text {backlog }}$ is small, the proposed enhanced algorithm can determine that the random data loss is caused by the high BER of the satellite link, and the value of CWND can be determined as follows:

$c w n d+=\sqrt{\rho} / 4 \times S M S S$ 
where $S M S S$ is the maximum segment size set by the sender. The slow start was divided into three sub-phases, in which CWND was adjusted in accordance with different network conditions. In the initial connection, CWND increased the network to be detected in the first sub-phase, especially when the network has entered a high load degree. The new connection detected the available bandwidth of the network to prevent degradation of network performance caused by the influx of massive data. The improved scheme adopted cwnd $+=\sqrt{\rho} / 4 \times S M S S$ for the increase in CWND in the initial stage. After the available bandwidth of the network was detected, the value of CWND was adjusted in accordance with the network condition. In the second subphase, the network condition was judged and detected by comparing CWND, threshold, the size of unacknowledged bytes, and the notification window. If the network is in good condition, the increase range can be appropriately extended. In the scheme, the CWND was $\sqrt{r} ¥ S M S S$. In the last subphase, the increase amplitude was decreased appropriately, and the setting of the initial stage was also adopted. The CWND was $c w n d+=\sqrt{\rho} / 4 \times$ SMSS accordingly, the network congestion caused by excessive increase rate could be avoided. The rwnd (receiver advertisement window) and flightsize (the total number of unacknowledged bytes in the network) are included in the information of the returned packet. The ssthresh is the slow start threshold.

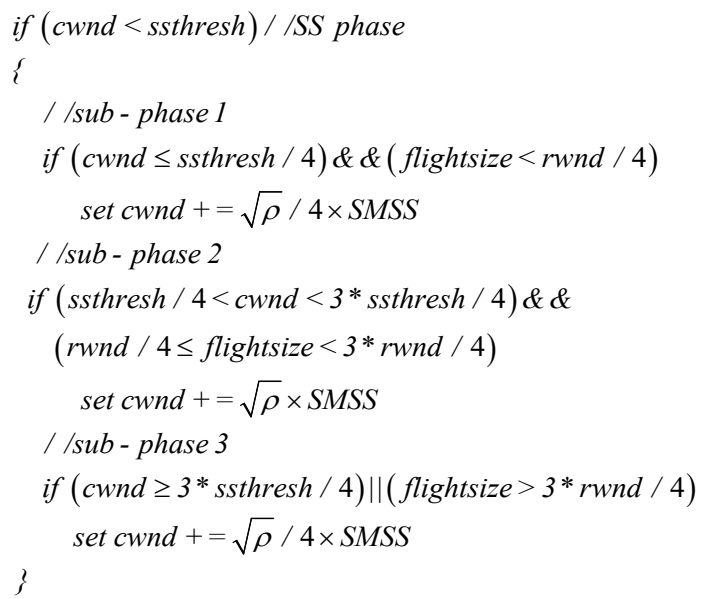

\subsection{Congestion avoidance}

In the detection of packet loss, the method of data backlog value was used to judge congestion packet loss in the network. The congestion degree was determined by comparing the data backlog with the threshold.

If the minimum RTT is only half of the current RTT, then the backlog value in the network is half of CWND. When the minimum RTT in the network is only one fourth of the current RTT, the network backlog value is threequarters of CWND. In accordance with the above packet loss detection, the more evident the difference between $R T T_{\min }$ and $R T T$ is, the worse the network state and the more serious the congestion will be. The backlog value can be calculated by storing a minimum RTT value at the sender of the data, which is relatively simple.

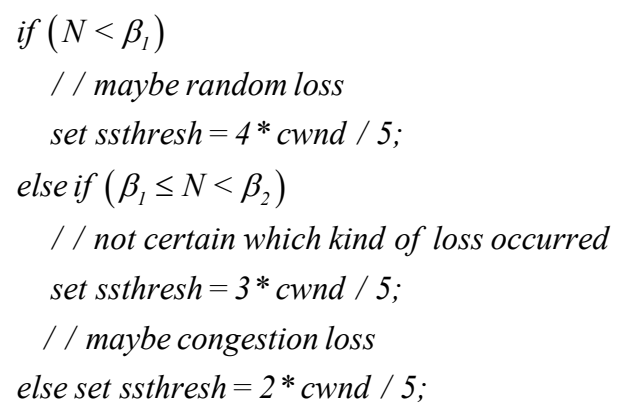

$\beta_{l}, \quad \beta_{2}$ are the adjusted thresholds. In a satellite network environment with massive random losses, the transmission rate of the improved algorithm is considerably enhanced compared with that of the traditional transmission control protocol.

\section{Result analysis and discussion}

The experimental simulation software is OPNET 14.5, and the simulation model is shown in Fig. 1. The data sender is the server, which connects to the gateway of the data receiver through a GEO satellite and connects to a client. The link rate between the server and gateway is $10 \mathrm{Mb} / \mathrm{s}$. The downlink (gateway to client) and uplink (client to gateway) rates in satellite networks are $2,048 \mathrm{~kb} / \mathrm{s}$ and 256 $\mathrm{kb} / \mathrm{s}$, respectively. In the experiment, the BER of the satellite link is $10^{-9}$ to $10^{-5}$. The data sender sends data to the client, and the data size is $50 \mathrm{MB}$. In the simulation, the main statistics are the response time of the client and the throughput of the satellite link. In the experiment, the cache size at the gateway is set to simulate the data backlog in the gateway. It is divided into two scenarios: the ground gateway has massive data backlog (the gateway cache is 37 500 bytes) and the ground gateway has minimal data backlog (the gateway cache is $16 \mathrm{M}$ bytes). The gateway cache is set to adjust the size of the backlog data. In addition to the classic TCP New Reno, the proposed algorithm also compares TCP Veno and TCP ADaLR. $\mathrm{r}$ is 15 , and $\beta_{1}$, $\beta_{2}$ are 3 and 6 , respectively.

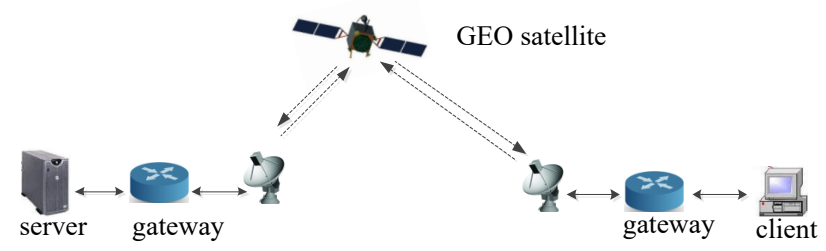

Fig. 1. Simulation model

\subsection{No massive data backlog in the ground gateway}

On the basis of the transmission factor in Subsection 3.1 and the slow start in Subsection 3.3, the satellite link throughput and the download response time of the proposed scheme without congestion in the satellite network are analyzed. Fig. 2 shows the download response time for different BERs under a large gateway cache. When BER changes from $10^{-9}$ to $10^{-7}$, the four different transmission schemes have similar download response time. In the case of a low BER, the probability of data loss caused by bit error is low. Therefore, the difference between the schemes of data retransmission and data loss discrimination is small, and a minimal influence on the download response time of four 
transmission schemes is induced. When BER changes from $10^{-7}$ to $10^{-6}$, the transmission schemes show evident differences on download response time. When BER changes from $10^{-6}$ to $10^{-5}$, the download response time increases dramatically. The download response time of Veno is slightly shorter than that of New Reno. The proposed scheme has shorter download response time. In the case of a high BER $\left(10^{-5}\right)$, the response time from long to short is as follows: TCP New Reno, TCP Veno, TCP ADaLR, and the proposed scheme.

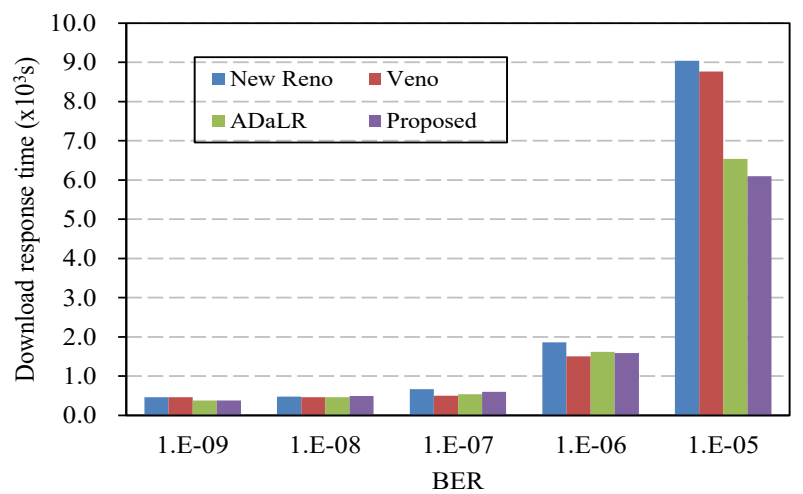

Fig. 2. Download response time for the scenario with a large gateway memory

Fig. 3 shows the throughput of a satellite link under different BERs in the case of a large gateway cache. As BER increases, the throughput of the four transmission schemes decreases gradually, indicating that the change in BER directly influences the throughput of satellite networks. The throughput of the proposed scheme is higher than three other transmission schemes. When BER changes from $10^{-9}$ to $10^{-5}$, the performance of New Reno is the worst. In the case of a low BER $\left(10^{-9}\right)$, the downlink utilization rate of the proposed scheme is $76 \%$, which is $6 \%$ higher than $70 \%$ of TCP ADaLR. When BER is $10^{-6}$, the downlink utilization rate of the proposed scheme is decreased to $16 \%$, but it is still 3\% higher than that of TCP ADaLR. A high BER causes serious data loss, which directly affects the utilization rate of satellite links. The throughput of the satellite link with TCP Veno and New Reno is low. The increase throughput of the two schemes is smaller than that of TCP ADaLR in the slow start, which has a great constraint on adapting to the long delay of the satellite link. Under high BER $\left(10^{-5}\right)$, the throughput of the satellite link of the proposed improved scheme is highest, which is nearly $20 \%$ higher compared with TCP ADaLR.

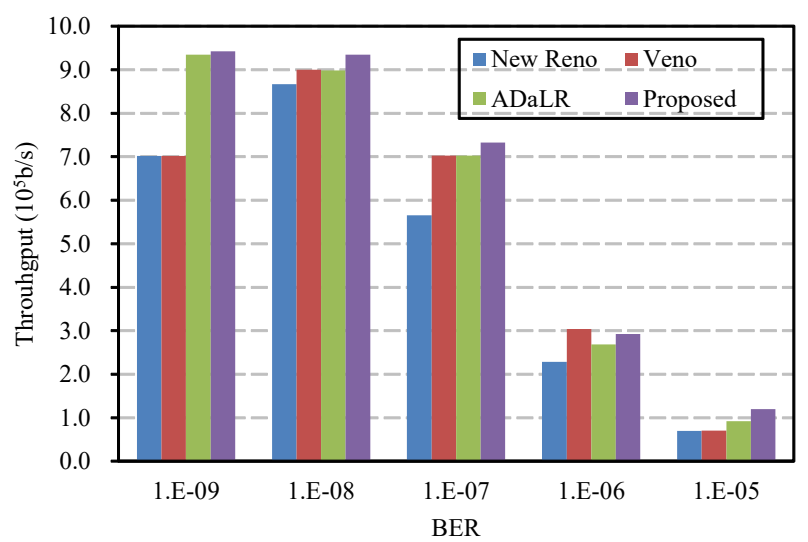

Fig. 3. Satellite link throughput for the scenario with a large gateway memory
On the whole, when the gateway cache is large, the download response time of the proposed scheme is smaller than TCP ADaLR, and the difference is obvious under a high BER. TCP Veno and TCP New Reno have longer download response time because of the conservative data increase for satellite links with long delay. Similarly, the proposed scheme has higher throughput than TCP ADaLR. TCP Veno and TCP New Reno have lower link throughput than TCP ADaLR.

\subsection{Massive data backlog in the ground gateway}

Based on the detection of packet loss mechanism in Subsection 3.2 and the congestion avoidance mechanism in Subsection 3.4, this subsection analyzes the satellite link throughput and download response time of the proposed scheme during congestion occurrence in the satellite network. Fig. 4 shows that when the gateway cache is small, the download response time changes at different BERs. The download response time of the four different transmission schemes has a minimal difference under a low BER $\left(10^{-9}\right.$ to $10^{-7}$ ), which is roughly consistent with the change trend in Fig. 4. Random data loss is less likely to be caused by the low BER, which exerts a limited effect on download response time. Therefore, the four schemes have a small difference in download response time. However, in the case of a high BER $\left(10^{-7}\right.$ to $\left.10^{-5}\right)$, especially when BER is $10^{-5}$, the download response time of the four transmission schemes is relatively different. The download response time of the proposed algorithm is approximately $7.542 \times 10^{3} \mathrm{~s}$, which is $15.6 \%$ less than the $8.936 \times 10^{3} \mathrm{~s}$ of TCP ADaLR. Under a high BER, data loss is caused by random loss and congestion of cache overflow.

TCP Veno and TCP New Reno schemes adopt a relatively conservative data increase for satellite links with long delay. The proposed scheme increases the throughput of satellite link by increasing the amount of data transmission. Based on the detection of packet loss mechanism the proposed scheme distinguishes the two types of data loss and reasonably adjusts the value of CWND in accordance with the reason for data loss. Consequently, the response time of download of the proposed scheme can be shortened compared with TCP New Reno, TCP Veno and TCP ADaLR.

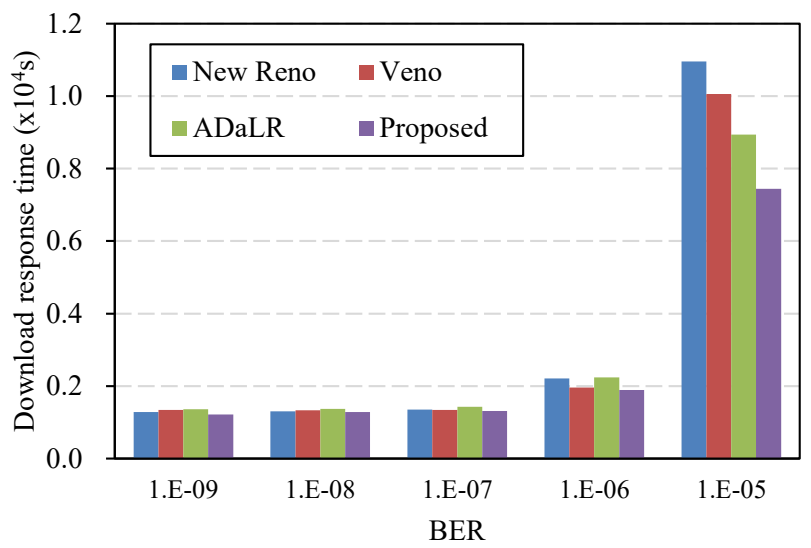

Fig. 4. Download response time for the scenario with a small gateway memory 


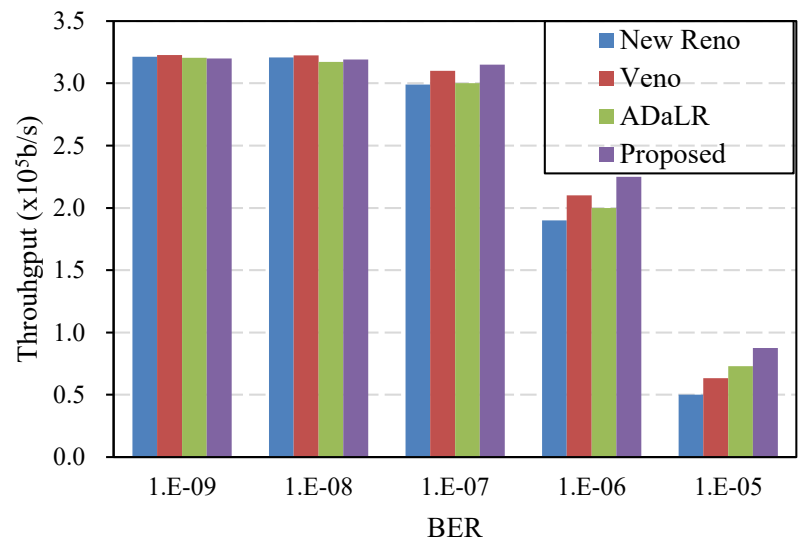

Fig. 5. Satellite link throughput for the scenario with a small gateway memory

Fig. 5 shows the change in the throughput of a satellite link under different BERs when the gateway cache is small. As the BER of the satellite link increases, the throughput of the link gradually decreases. Compared with Fig. 3, Fig. 5 depicts that the four schemes show a less obvious difference in the satellite link throughput under a low BER. In the case of a high BER $\left(10^{-6}\right.$ to $\left.10^{-5}\right)$, the throughput of the proposed algorithm is significantly improved compared with three other transmission schemes, and the discrimination is further increased. At a high BER $\left(10^{-5}\right)$, the satellite link throughput of TCP Veno is higher than that of TCP New Reno. The satellite link throughput of the proposed scheme is highest, which is nearly higher $13 \%$ compared with that of TCP ADaLR. The figure demonstrates that the satellite link throughput of the proposed scheme is higher than three other schemes when the gateway cache is small.

Under a small gateway cache, the download response time of the proposed scheme is generally shorter than TCP ADaLR. When BER is high, the difference degree further increases because the proposed scheme optimizes CWND and distinguishes packet loss. The link throughput also shows an evident difference. Nevertheless, TCP Veno and TCP New Reno schemes still adopt a relatively conservative data increase for satellite links with long delay, and the data loss is determined as congestion loss. Consequently, the transmission performance is substantially decreased, resulting in increased download response time and decreased throughput of the satellite link.

\section{Conclusions}

To improve the performance of transmission control algorithms for satellite networks, the proposed algorithm increases the amount of transmission data and distinguishes data packet loss. The two improvement aspects are aimed at the long delay and high BER of satellite links. In the simulation experiments, TCP ADaLR, TCP Veno, and TCP New Reno were compared with the proposed algorithm. The following conclusions were drawn:

(1) Compared with the three congestion control algorithms, the proposed scheme improve the throughput and download response time of satellite links.

(2) When the cache of the gateway is small, the proposed scheme can distinguish random and congestion packet losses and its performance had improved obviously.

(3) In the fast recovery, if the data loss is evaluated to be caused by random loss, then the threshold is optimized compared with the traditional TCP New Reno and the proposed algorithm can rapidly improve the throughput of the satellite network.

In this study, a new optimization scheme was proposed by combining experiment and theoretical study. The CWND of slow start and congestion avoidance was redesigned by introducing a transmission factor and a congestion avoidance mechanism. The transmission mechanism can improve the transmission performance of satellite networks, which has reference significance to the follow-up study of satellite network transmission control. Owing to the lack of an actual field test environment, future study will modify the proposed scheme in combination with actual test; hence, the transmission mechanism of satellite networks can be understood more thoroughly.

\section{Acknowledgements}

This work was supported by the Hunan Provincial Natural Science Foundation (Grant No. 2020JJ4557), the Scientific Research Fund of Hunan Provincial Education Department (Grant Nos. 20B519, 19B512 and 19A446), and the National Natural Science Foundation of China (Grant No. 61961014).

This is an Open Access article distributed under the terms of the Creative Commons Attribution License

\section{References}

1. Chini, P., Giambene, G., Kota S., "A survey on mobile satellite systems". International Journal of Satellite Communications and Networking, 28(1), 2010, pp.29-57.

2. Ghani, N., Dixi,t S., "TCP/IP enhancements for satellite networks". IEEE Communications Magazine, 37(7), 1999, pp.64-72.

3. Zhu, X., Jiang, C., Kuang, L., Ge, N., Guo, S., Lu, J., "Cooperative transmission in integrated terrestrial-satellite networks". IEEE network, 33(3), 2019, pp.204-210.

4. Lin, Z., Lin, M., Wang, J. B., Huang, Y., Zhu, W. P., "Robust secure beamforming for $5 \mathrm{G}$ cellular networks coexisting with satellite networks". IEEE Journal on Selected Areas in Communications, 36(4), 2018, pp. 932-945.

5. Jia, X., Lv, T., He, F., Huang, H., "Collaborative data downloading by using inter-satellite links in LEO satellite networks". IEEE Transactions on Wireless Communications, 16(3), 2017, pp.15231532.

6. Marchese, M., Moheddine, A., Patrone, F., "IoT and UAV integration in 5G hybrid terrestrial-satellite networks". Sensors, 19(17), 2019, pp. 3704-3733.
7. De Gaudenzi, R., Del Rio Herrero, O., Gallinaro, G., Cioni, S., Arapoglou, P. D., "Random access schemes for satellite networks, from VSAT to M2M: a survey". International Journal of Satellite Communications and Networking, 36(1), 2018, pp.66-107.

8. Wang, J., Pham, K., "Design of Nonlinear Control for Active Queue Management in TCP Satellite Communication Networks". In: 2020 IEEE Aerospace Conference, Big Sky, USA: IEEE, 2020, pp. 1-9.

9. Abdelsalam, A., Luglio, M., Roseti, C., Zampognaro, F., "TCP connection management through combined use of terrestrial and satellite IP-Based links". In: 2017 40th International Conference on Telecommunications and Signal Processing (TSP), Barcelona, Spain: IEEE, 2017, pp. 37-42.

10. Giambene, G., Luong, D. K., Le, V. A., de Cola, T., Muhammad, M., "Transport layer performance combining multipath and network coding in mobile satellite networks". International Journal of Satellite Communications and Networking, 35(6), 2017, pp.583603. 
Liang Zong, Yudan Ning, Yong Bai, Chenglin Zhao, and Gaofeng Luo/

Journal of Engineering Science and Technology Review 13 (6) (2020) 74 -80

11. Bisu, A. A., Purvis, A., Brigham, K., Sun, H., "A Framework for End-to-End Latency Measurements in a Satellite Network Environment". In: 2018 IEEE International Conference on Communications (ICC), Kansas City, USA: IEEE, 2018, pp. 1-6.

12. Bisu, A. A., Gallant, A., Sun, H., Brigham, K., Purvis, A., "Experimental Performance Evaluation of TCP Over an Integrated Satellite-Terrestrial Network Environment". In: 2019 15th International Wireless Communications \& Mobile Computing Conference (IWCMC), Tangier, Morocco: IEEE, 2019, pp. 781786.

13. Saedi, T., El-Ocla H., "TCP CERL+: revisiting TCP congestion control in wireless networks with random loss". Wireless Networks, 2020, pp.1-18.

14. Liu, Z., Yang, Y., Zhao, W., Zhang, M., Wang, Y., "Research on Congestion Control Over Wireless Network with Delay Jitter and High Ber”. In: International Conference on Computer Engineering and Networks. Singapore: Springer, 2020, pp. 1514-1521.

15. Nie, X., Zhao, Y., Li, Z., Chen, G., Sui, K., Zhang, J.,Ye, Z., Pei, D., "Dynamic TCP initial windows and congestion control schemes through reinforcement learning". IEEE Journal on Selected Areas in Communications, 37(6), 2019, pp.1231-1247.

16. Polese, M., Chiariotti, F., Bonetto, E., Rigotto, F., Zanella, A., Zorzi, M., "A survey on recent advances in transport layer protocols". IEEE Communications Surveys \& Tutorials, 21(4), 2019, pp.3584-3608.

17. Zhang, T., Mao, S., "Machine learning for end-to-end congestion control". IEEE Communications Magazine, 58(6), 2020, pp.52-57.

18. Li, N., Deng, Z., Zhu, Q., Du, Q., “AdaBoost-TCP: A Machine Learning-Based Congestion Control Method for Satellite Networks". In: 2019 IEEE 19th International Conference on Communication Technology (ICCT), Xi'an, China: IEEE, 2019, pp. 1126-1129.

19. Deutschmann, J., Hielscher, K. S., German, R., "Satellite internet performance measurements". In: 2019 International Conference on Networked Systems (NetSys). Munich, Germany: IEEE, 2019, pp. 14.

20. Giambene, G., Kota, S., Pillai, P., "Satellite-5G integration: A network perspective “. IEEE Network, 32(5), 2018, pp.25-31.
21. Ishii, A., Usuki, Y., Nakagawa, K., Zabir, S. M. S., Utsumi, S., “A New Real-Time Rate Control Friendly with TCP Hybla over Heterogeneous Networks". In: 2019 IEEE 90th Vehicular Technology Conference (VTC2019-Fall), Hawaii, USA: IEEE, 2019, pp. 1-7.

22. Secchi, R., Mohideen, A. C., Fairhurst, G., "Performance analysis of next generation web access via satellite". International Journal of Satellite Communications and Networking, 2018, 36(1), pp. 2943.

23. Quadrini, M., "Performance evaluation of a QUIC-based proxy architecture over a hybrid satellite-terrestrial backhaul network". In: 2019 International Symposium on Advanced Electrical and Communication Technologies (ISAECT), Rome, Italy: IEEE, 2019, pp. 1-5.

24. Utsumi, S., Zabir, S. M. S., Usuki, Y., Takeda, S., Shiratori, N., Kato, Y., Kim, J., A new analytical model of TCP Hybla for satellite IP networks". Journal of Network and Computer Applications, 124, 2018, pp.137-147.

25. Liu, J., Han, Z., Li, W., "Performance Analysis of TCP New Reno Over Satellite DVB-RCS2 Random Access Links". IEEE Transactions on Wireless Communications, 19(1), 2019, pp.435446.

26. Fraire, J. A., Céspedes, S., Accettura, N., "Direct-To-Satellite IoTA Survey of the State of the Art and Future Research Perspectives". In: International Conference on Ad-Hoc Networks and Wireless, Luxembourg, Luxembourg: Springer, 2019, pp. 241258.

27. Shijie, G., Yueqiu, J., Qixue, G., Guo, Q. C., "Research on Congestion Control Algorithm of Satellite Network Based on Forword Direction Delay “, Acta Armamentarii, 39(5), 2018, pp.945-951.

28. Haiguang X., Lianghui D., Feng Y., “An Enhancement of Multipath TCP for Heterogeneous Satellite Networks". Computer Simulation. 37(7), 2020, pp.95-99.

29. Fu, C. P., Lu, W., Lee, B. S., "TCP Veno revisited". In: GLOBECOM'03. IEEE Global Telecommunications Conference, San Francisco, USA: IEEE, 2003, pp. 3253-3258. 